\title{
Ortho-topolin riboside induced cell apoptosis through ERS pathway and inhibited DNMT1 activity in acute myeloid leukemia cells.
}

\author{
Li Wang ${ }^{1 \#, ~ Y a n H o n g ~ Z h a o " ~}{ }^{2 \#}$, Jiao Cheng ${ }^{1}$, FanLin Lin ${ }^{1}$, YingYing Deng ${ }^{1}$, Changhao Cui ${ }^{*}$ \\ ${ }^{1}$ School of Life and Medicine, Dalian University of Technology, Pan Jin, Liaoning, P. R China \\ ${ }^{2}$ Department of Hematology, First Affiliated Hospital, Harbin Medical University, Harbin, Heilongjiang, P. R China \\ \#These authors have equally contributed to this study.
}

\begin{abstract}
We previously demonstrated that ortho-topolin riboside (oTR), a naturally occurring phytohormone, has potential anticancer effects via the mitochondrial apoptotic pathway in acute promyelocytic leukemia HL-60 cells and hepatocellular carcinoma SMMC-7721 cells. In the present study, we showed that oTR inhibited the of acute myeloid leukemia (AML) U937 cells. Cellular adenosine transporte uptake of oTR but not adenosine receptors was involved in the growth inhibition. We also found oTR induced cell apoptosis through the Endoplasmic Reticulum Stress (ERS) pathway, as evidenced by the upregulation of the ERS regulator glucose regulated protein 78. Exposure of U937 cells to oTR upregulated the surface marker $\mathrm{CD11b}$, reduced the nuclear cytoplasmic ratio, and altered the horseshoe shape of nuclei, as determined by Wright-Giemsa staining. Further we found that oTR as an effective inhibitor decrease the DNA methyltransferase 1 (DNMT1) activity in a dose-dependent manner. Dock study showed that oTR can dock to the putative pocket of the DNMT1. We concluded that oTR induced apoptosis, promoted the cell differentiation and inhibited DNMT1 activity of U937 cells, suggesting its potential as a therapeutic agent for the treatment of AML.
\end{abstract}

Keywords: Ortho-topolin riboside, Endoplasmic reticulum stress, DNMT1, U937cells.

Accepted on March 26, 2018

\section{Introduction}

Acute Myeloid Leukemia (AML) consists of a group of heterogeneous diseases with abnormally active proliferation of hematopoietic precursors, which block normal hematopoiesis, causing neutropenia and anemia [1]. Efforts to improve standard cytotoxic chemotherapy, the current approach to AML treatment, have been unsuccessful, underscoring the need to develop new chemotherapeutic agents capable of effectively removing or reducing leukemic blasts in AML [2].

Cytokinins, as important purine derivatives with phytohormone activity, play key roles in the regulation of plant growth and differentiation. This led to the postulation that they could also affect growth and differentiation in animals and have potential utility for treating human diseases that involve dysfunctional cell proliferation and/or differentiation [3]. Cytokinin ribosides (N6-substituted adenosine derivatives) are nucleoside analogues that have shown anticancer activity both in vitro and in vivo in mammals [3-11].

6-(2-hydroxybenzylamino)-9-D-ribofuranosylpurine or orthotopolin riboside (oTR, Figure 1) is a naturally occurring riboside form of cytokinins that is present at micromolar concentrations in poplar leaves after daybreak. oTR has shown exceptional cytotoxic activity against NCI60 cell lines when compared with other cytokinin ribosides $[3,12]$. However, the detailed molecular mechanism underlying its anticancer effect has not been elucidated, and little is known about the effect of differentiation on carcinoma cells.

Epigenetic gene silencing is an important mechanism that results in loss of gene expression and that mediates, along with genetic mutations, the initiation and progression of human cancer. Some reports showed that nucleoside analogs can reverse epigenetic silencing in cancer [13].

In our previous studies, we demonstrated that oTR exerts growth inhibitory and apoptotic effects on the human hepatocellular carcinoma cell line SMMC-7721 and acute promyelocytic leukemia cell line HL-60 through the mitochondrial apoptotic pathway. In the present study, we evaluated the growth inhibitory effects of oTR on human AML U937 cells mediated by the induction of apoptosis and differentiation. Our results showed that oTR inhibited the proliferation of U937 cells by inducing apoptosis via the endoplasmic reticulum stress (ERS) pathway, as evidenced by the increased level of glucose regulated protein 78 (GRP78). Moreover, flow cytometry analysis showed that oTR treatment upregulated the expression of the cell surface marker CD11b, 
and Wright-Giemsa staining showed that it promoted the differentiation of U937 cells towards a mature granulocyte lineage. Furthermore we found that oTR decreased the DNA methyltransferase 1(DNMT1) activity in a dose-dependent manner. Dock study showed that oTR can dock to the putative pocket of the DNMT1. These data indicated that oTR, as a natural nucleoside analogue compound, may be a potential alternative therapeutic choice of targeting DNA methylation for the treatment of human acute leukemia.

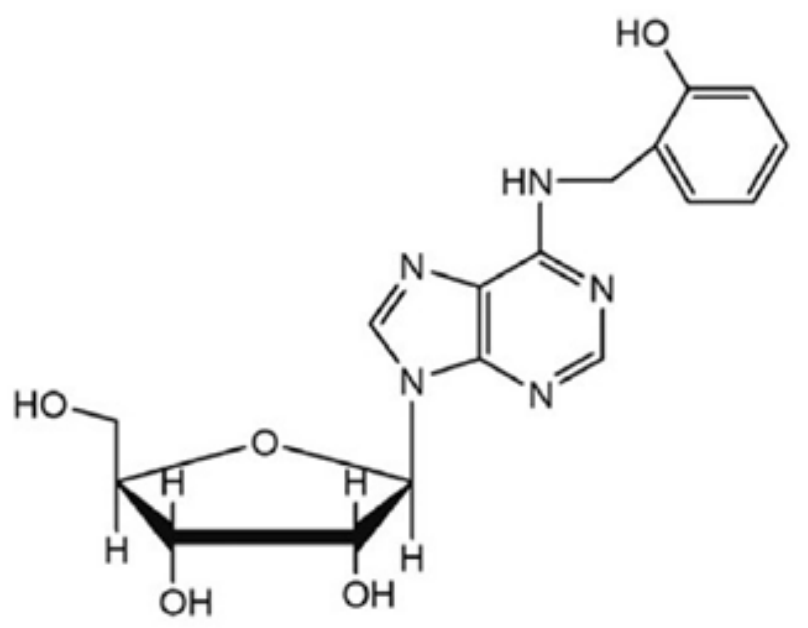

Figure 1. Structure of ortho-topolin riboside (oTR). Molecular weight $=373.4$.

\section{Materials and Methods}

\section{Reagents}

oTR, with a purity $>99 \%$, was purchased from OlChemim Ltd. (Czech Republic). The compound was dissolved in dimethyl sulfoxide (DMSO), stored at $4^{\circ} \mathrm{C}$, and diluted to the required concentration prior to use. The maximal final concentration of DMSO in the culture medium was below 0.2\%. DMEM with L-glutamine and sodium bicarbonate, Fetal Bovine Serum (FBS) were purchased from Hyclone (Logan, UT, USA). Cell Counting Kit-8 was purchased from Sangon Biotech (Shanghai, China). Wright-Giemsa staining solution was purchased from Sigma-Aldrich Corporation (St. Louis, MO, USA). Anti-Human CD11b-PE was purchased from eBioscience (San Diego, CA, USA), Antibodies against GRP78 and $\beta$-actin were purchased from Santa Cruz Biotechnology Inc. (Santa Cruz, CA, USA). Annexin V-FITC Apoptosis Kit, Propidium Iodide (PI) was purchased from the Beyotime Institute of Biotechnology (Beijing, China). A1 adenosine receptor antagonist 1, 3-Dipropyl-8cyclopentylxanthine (DPCPX), A2a adenosine receptor antagonist 7-(2-phenylethyl)-5-amino-2-(2-furyl)pyrazolo-(4, 3-e)-1,2,4-triazolo (1,5-c) pyrimidine (SCH58261), A2b adenosine receptor antagonist 8-(4-(((4Cyanophenyl) carbamoylmethyl) oxy) phenyl)-1, 3-di (npropyl) xanthine hydrate (MRS1754), A3 adenosine receptor antagonist 3-Ethyl-5-benzyl-2-methyl-4- phenylethynyl-6-phenyl-1, 4-( \pm )-dihydropyridine-3, 5dicarboxylate (MRS1191) were purchased from Sigma (St. Louis, USA). EpiQuik DNA Methyltransferase Activity Assay Kit and EpiQuik DNA Methyltransferase 1 Activity/Inhibitor Screening Assay Kit were purchased from Epigentek Inc.

\section{Cell culture}

U937 cells were obtained from the Cell Bank of the Chinese Academy of Sciences (Shanghai, China). The cells were grown in culture plates containing DMEM medium supplemented with $10 \%$ fetal bovine serum at $37^{\circ} \mathrm{C}$ and $5 \% \mathrm{CO}_{2} / 95 \%$ air.

\section{Cell viability assay}

Cell viability was assayed in 96-well flat-bottomed plastic microplates. Cells were seeded at a density of 5000 cells per well with a series of concentrations $(0-50 \mu \mathrm{M})$ for $72 \mathrm{~h}$. Cell viability was analyzed using the Cell Counting Kit-8 (CCK-8); $10 \mu \mathrm{l}$ of CCK-8 solution was added to each well before incubation for further $3 \mathrm{~h}$ at $37^{\circ} \mathrm{C}$. After incubation, the absorbance was measured at $450 \mathrm{~nm}$ using a microplate reader (3001, Thermo Scientific, Finland). The growth inhibition rate was expressed as (control absorbance-sample absorbance)/ control absorbance $\times 100 \%$.

\section{Western blot assay}

Western blotting was used as previously described [14]. After oTR treatment for the time indicated, the level of GRP78 protein was detected by enhanced chemiluminescence and exposed to Kodak X-OMAT autoradiographic film (Kodak, Hemel Hempstead, UK).

\section{Apoptosis analysis by Annexin V-FITC and PI staining}

Harvested cells were washed three times with ice-cold phosphate-buffered saline (PBS), suspended in binding buffer and stained with AnnexinV-FITC and (or) phosphatidylinositol (PI) at room temperature for $30 \mathrm{~min}$ in the dark. Cells were then analyzed by flow cytometry (FACS Calibur; Becton Dickinson, USA).

\section{Measurement of CD11b protein expression}

CD11b protein expression at the cell surface was analyzed by flow cytometry. After treatment with oTR, the cells were collected and washed three times with PBS which fixed with $0.2 \%$ FBS. Cells were incubated for $15 \mathrm{~min}$ at room temperature with blocking antibody, and then incubated with anti-Human CD11b-PE antibody for $30 \mathrm{~min}$ at $4{ }^{\circ} \mathrm{C}$ in the dark. Cells were then washed and analyzed by flow cytometry (FACSCalibur, Becton Dickinson, USA).

\section{Wright-Giemsa staining}

After $72 \mathrm{~h}$ treatment with oTR, cells were collected and washed with PBS. The slides were fixed with methanol and stained with Wright-Giemsa staining solution for $20 \mathrm{~min}$, 
rinsed with distilled water, air-dried and observed using a microscope (Leica, DMI4000B, and Germany).

\section{Assay for DNMT and DNMT1 activity}

Nuclear extracts of treated cells were prepared using a nuclear extraction reagent following the manufacturer's instructions. The DNMT activity was determined in the nuclear extracts using the EpiQuik DNA Methyltransferase Activity Assay Kit (Epigentek Inc.). Recombinant DNMT1 was obtained from Active Motif. DNMT1 enzyme was incubated with different concentrations of oTR, and the DNMT1 activity was determined using the EpiQuik DNA Methyltransferase 1 Activity/Inhibitor Screening Assay Kit (Epigentek Inc.) with a microplate reader at $450 \mathrm{~nm}$.

\section{Molecular modelling}

The LibDock method in Discovery Studio 2016 software was carried out to investigate the binding modes of DNMT1 when acting with oTR. The X-ray crystal structure of DNMT1 (PDB code 3AV6) of AdoMet was prepared by removing water of crystallization and adding hydrogen atoms. The ligand was extracted from the crystal structure to generate the prototype molecule. The Structures of oTR was generated with ChemBioDraw Ultra 14.0 and energy was minimized with the CHARMm program. Then flexible amino acid and binding sphere was prepared. The binding sphere is selected as a radius of $9 \mathrm{~A}^{\circ}$, with the center coordinates of $\mathrm{X}: 76.728727, \mathrm{Y}$ : 12.895909 and Z: 39.989364. Then oTR was docked into DNMT1 by using Libdock, and then an automatically achieved docking mode was obtained with scoring results about the ligand-receptor combination, which could be used for DNMT1 inhibitory activity prediction.

\section{Statistical analysis}

Results are expressed as the mean \pm SD. The student's t-test and one-way ANOVA were used to compare the test and control values. ${ }^{*} \mathrm{p}<0.05,{ }^{* *} \mathrm{p}<0.01$ were considered statistically significant compared to the control.

\section{Results}

\section{Effect of oTR on U937 cell proliferation}

Cells exposed to increasing concentrations of oTR $(0.1,0.5,1$, 5 and $10 \mu \mathrm{M}$ ) for $72 \mathrm{~h}$ showed a decrease in the total number of cells compared with that in the untreated control (Figure 2A). This dose-dependent inhibitory effect on cell proliferation was also observed using the CCK-8 assay, which showed that the rate of cell growth inhibition increased significantly from $5.3 \%$ to $8.3 \%, 29.5 \%, 69.5 \%$, and $71.8 \%$ with increasing concentrations of oTR within the range of 0.1-10 $\mu \mathrm{M}$ (Figure 2B).
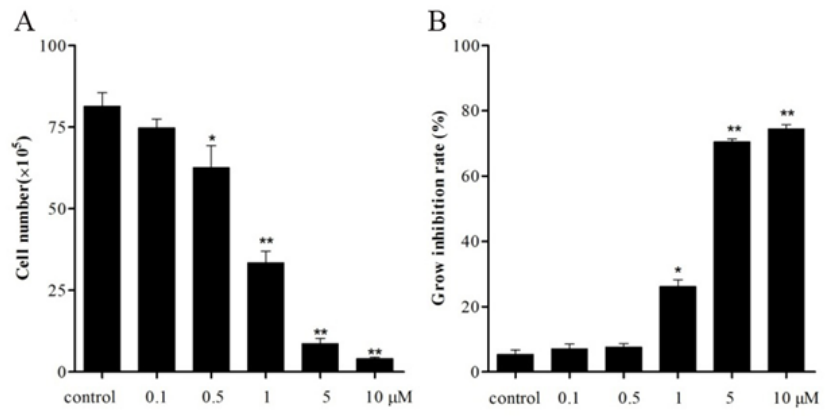

Figure 2. Effects of oTR on U937 cells. Cells were incubated with vehicle (0.2\% DMSO). (A) Cell numbers were counted after $72 \mathrm{~h}$ of treatment with increasing concentrations of oTR by hemocytometer; (B) Cell viability was analyzed by the CCK-8 assay after $72 \mathrm{~h}$ of treatment with increasing concentrations of oTR. The values are expressed as the mean $\pm S D$ from three individual experiments. ${ }^{*} p<0.05,{ }^{* *} p<0.01$ versus control.

\section{oTR induces apoptosis in $\mathrm{U937}$ cells}

To further investigate the mechanisms underlying oTR-induced cell apoptosis, Treated U937 cells were detected by flow cytometry after staining with Annexin V-FITC and PI (Figure 3). The results showed that oTR significantly induced apoptosis in a time-dependent manner. Apoptotic cells (FL1+/ FL3-) increased from $1.87 \%$ in the control group to $17.25 \%$ in the oTR-treated group.

\section{No implication of adenosine receptors in oTR induced cell death}

We have found the apoptosis was significantly suppressed by the adenosine transporter inhibitor dipyridamole before [14]. Our next question to address is whether oTR-induced cell death is mediated via adenosine receptors or not, we treated cells with antagonists of A1 A2a A2b and A3 adenosine receptors. The results showed that the oTR action on cell viability was not inhibited by these adenosine receptors (Figure 4). This would rule out the possibility for the implication of adenosine receptors in oTR induced cell death.

\section{oTR induced endoplasmic reticulum (ER) stress- related response}

ER-mediated apoptosis is activated by a failed Unfolded Protein Response (UPR) related to ER stress-related proteins [15]. Changes in the levels of ER stress-related proteins in response to oTR treatment were investigated by western blot analysis of glucose-regulated protein $78 \mathrm{kDa}$ (GRP78) after treatment with $10 \mu \mathrm{M}$ oTR for $24 \mathrm{~h}$. The results showed that oTR remarkably increased the levels of the GRP78 protein compared with that in the control group (Figure 5). 

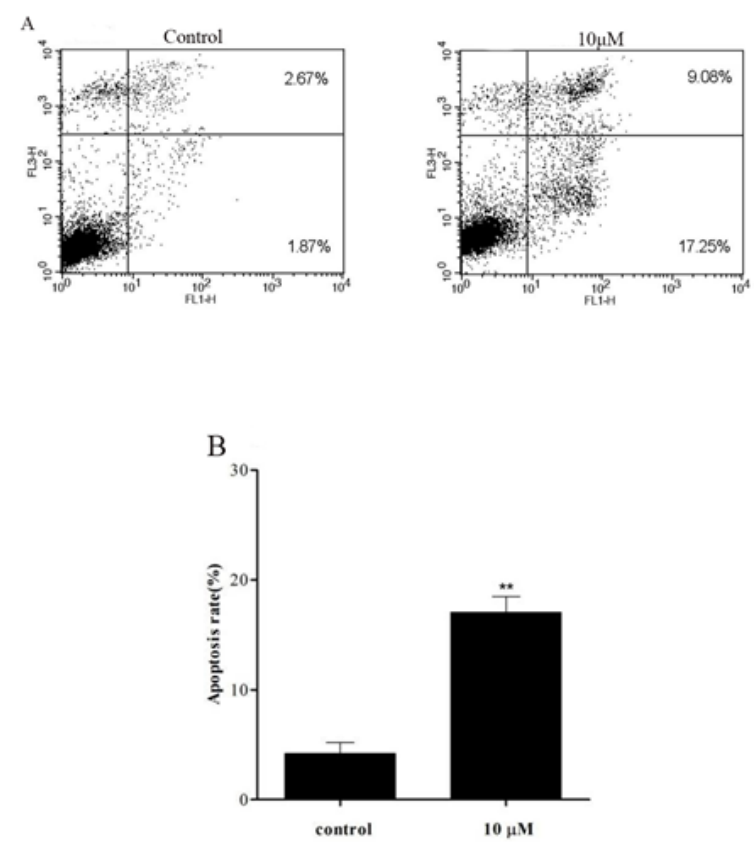

Figure 3. oTR induces apoptosis in U937 cells. (A) Apoptosis of U937 cells treated with $10 \mu \mathrm{M}$ oTR was determined after $24 \mathrm{~h}$ of treatment by Annexin V-FITC (FL1) and PI (FL3) staining and flow cytometry; (B) The values are expressed as the mean $\pm S D$ from three individual experiments. ${ }^{* *} P<0.01$ versus control.
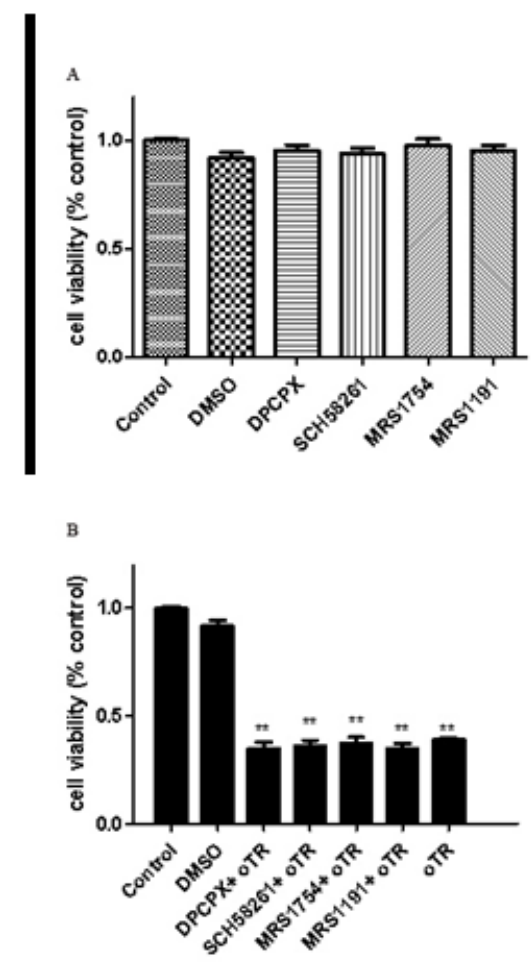

Figure 4. (A) Cell viability following treatment with different adenosine receptor antagonists $(5 \mu M)$ alone for $4 d$; (B) Cell viability following treatment with oTR $(1.5 \mu M)$ alone or in combination with different adenosine receptor antagonists for $4 d$. Data are represented as mean $\pm S D$ from three independent experiments ${ }^{* *} p<0.01$ compared to the control.

\section{Differentiation assay}

Differentiation therapies play important roles in leukemia treatment. We therefore investigated the differentiation effects induced by oTR by detecting the expression of CD11b, a marker for granulocytic differentiation. The results showed that oTR upregulated the expression of CD11b in U937 cells from $2.8 \%$ to $32.5 \%$ (Figure 6 ). This indicated that oTR induced the differentiation of U937 cells into mature granulocytes.
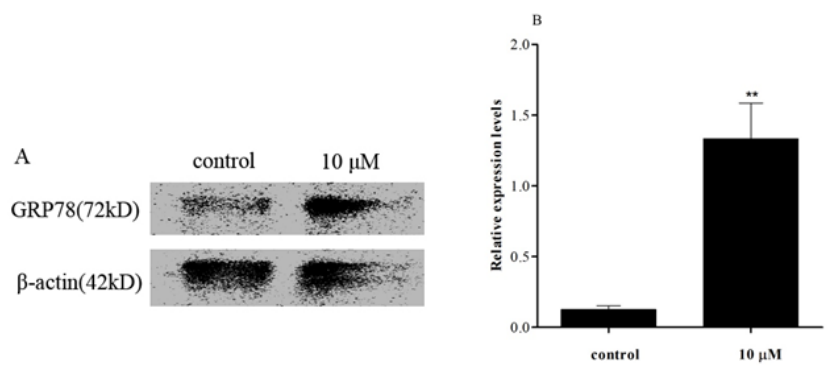

Figure 5. (A) Western blot analysis of GRP78 protein expression after treatment with vehicle (0.2\% DMSO) or $10 \mu M$ oTR for $24 h$; (B) Results were quantified by densitometric analyses of the bands from (A) and normalized to $\beta$-actin protein levels. The values are expressed as the mean $\pm S D$ from three individual experiments. ${ }^{* *} P<0.01$ versus control.
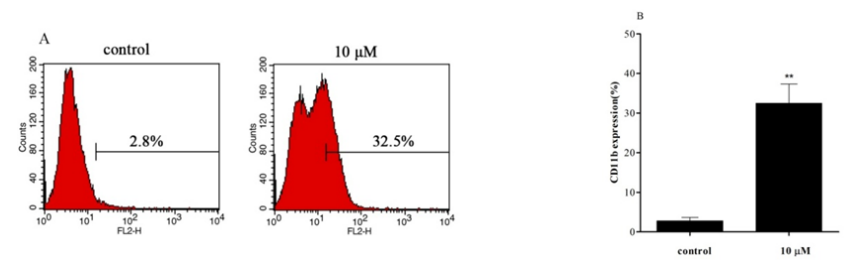

Figure 6. Effect of oTR on the expression of CD11b in U937 cells. (A) $C D 11 b$ protein expression at the cell membrane was analyzed by flow cytometry. The positive rate of CD11b-PE cells significantly increased after treatment with $10 \mu M$ oTR for $48 \mathrm{~h}$; (B) The values are expressed as the mean $\pm S D$ from three individual experiments. ${ }^{* *} P<0.01$ versus control.

\section{Wright-Giemsa staining}

The effect of oTR on inducing differentiation was confirmed by morphologic analysis using Wright Giemsa staining. Untreated U937 cells, as highly malignant cells, were round with large and round nuclei and a sparse cytoplasm. Treatment with oTR reduced the nuclear cytoplasmic ratio and altered the horseshoe morphology of nuclei (Figure 7).

\section{Assay for DNMT and DNMT1 activity}

Because DNMTs catalyze the process of DNA methylation, we assessed whether oTR changed the DNMT activity in U937 cells. Our study found that oTR decreases the DNMT activity in a dose-dependent manner in U937 cells (Figure 8A). Then we tested the inhibitory of DNMT1 treated by oTR, and the data (Figure 8B) showed that oTR is an effective DNMT1 inhibitor that can inhibit the DNMT1 activity in a dosedependent manner. 


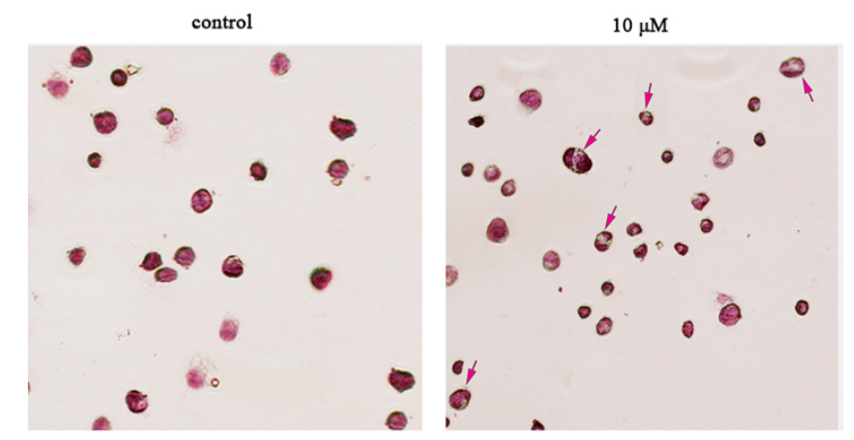

Figure 7. Effects of oTR on the morphology of U937cells. Cells were incubated with $10 \mu M$ oTR or vehicle (0.2\% DMSO) as a positive control for $48 \mathrm{~h}$, and morphological changes were observed by phase contrast microscopy after staining with Wright-Giemsa. The arrows indicate the cells with horseshoe shaped nuclei.
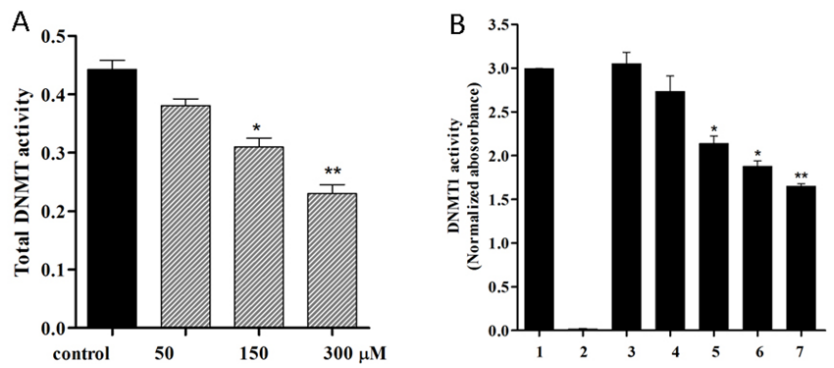

Figure 8. A) Effect of oTR treatment for 48 hours on the DNMT activity in U937 cells. B) DNMT1 activity assay in the presence of different concentration of oTR. 1: DNMT1; 2: $0.1 \mu M$ oTR; 3: $0.1 \mu M$ oTR+DNMT1; 4: $0.5 \mu M$ oTR+DNMT1; 5: $1 \mu$ MoTR+DNMT1; 6: 5 $\mu M o T R+D N M T 1 ; 7: 10 \mu M o T R+D N M T 1$.

\section{Molecular modelling}

The LibDock method in Discovery Studio 2016 software was carried out to investigate the binding modes of DNMT1 when acting with oTR. As shown in Figures 9A and 9B, oTR docked to the putative cytosine pocket with the pretty much the same LibDock Source compared with natural ligand Sadenosylmethionine (AdoMet). This pocket is the active region that facilitates binding with methylated DNA, a process that is required for subsequent methylation.
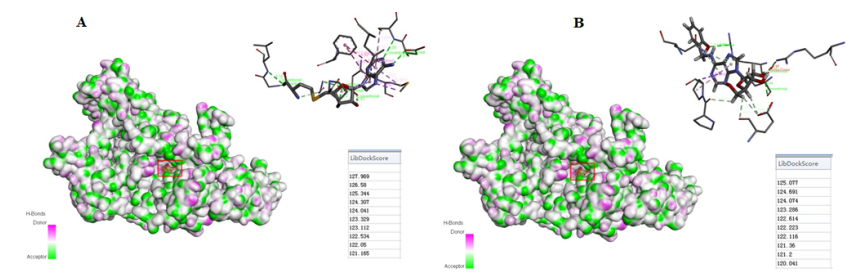

Figure 9. A) The binding modes of AdoMet with DNMT1 and LibDock Score; B) The binding modes of oTR with DNMT1 and LibDock Score.

\section{Discussion}

Leukaemia's are malignant blood diseases characterized by uncontrolled overproduction of hematopoietic progenitors or terminally differentiated leukocytes. AML is the most common adult acute leukemia [16]. Many anticancer agents have been reported to inhibit the growth of leukemia cells through the induction of apoptosis and/or differentiation [17]. Therefore, understanding the pathways and mechanisms of apoptosis and differentiation induced by novel therapeutic agents is crucial to improve the outcome of patients with AML.

In the present study, we showed that the plant hormone oTR significantly inhibited the proliferation of U937 AML cells, as indicated by the decrease in viability and cell number in response to treatment with oTR (Figure 2). Apoptosis was detected by flow cytometric analysis after staining with Annexin V-FITC and PI. As shown in Figures 3, oTR significantly increased the accumulation of apoptotic U937 cells (FL1: Annexin V+/PI-).

In animals, adenosine receptors play an important role in the regulation of a wide range of cellular and tissue functions in nervous, cardiovascular, gastrointestinal, respiratory and immune systems [18]. So we questioned whether oTR-induced cell death is mediated via adenosine receptors or not. We treated cells with antagonists of A1 A2a A2b and A3 adenosine receptors. The results showed that the oTR action on cell viability was not inhibited by these adenosine receptors (Figure 4). These results explain that extracellular oTR induces U937cell death by the adenosine transporter but not adenosine receptors.

Apoptosis pathways can be initiated via membrane proteins, mitochondria, or the ER. If the apoptosis pathway induced by a particular anticancer agent is identified, the anticancer effect can be maximized by combination chemotherapy aimed at activating several apoptosis pathways [19]. There are three types of signals that activate cell apoptosis: membrane protein signals, mitochondrial signals, and ER signals $[20,21]$. In our previous study, we demonstrated that oTR has potential anticancer effects mediated by mitochondrial signals in HL-60 cells. In the present study, we investigated whether ER signals are involved in the pro-apoptotic effect of oTR on U937 cells.

The ER is an essential organelle that regulates the synthesis and post-translational modifications of secretory and membrane proteins [22], Endoplasmic Reticulum Stress (ERS) is a condition that is accelerated by the accumulation of unfolded/misfolded proteins after a disturbance in the ERQC (ER quality control system) owing to a variety of physiological and pathological phenomena [23]. In mammals, there are three arms in the unfolded protein response (UPR) that involve distinct ER-transmembrane proteins, namely activating transcription factor- 6 , inositol-requiring enzyme- $1 \alpha$, and protein kinase RNA-like endoplasmic reticulum kinase, which act as ER stress sensors. Under normal conditions, these sensors are kept in an inactive form through their physical association with the master regulator of UPR, the glucoseregulated protein 78 (GRP78) chaperone [24,25]. However, abnormal external stimuli can activate ER stress, leading to the dissociation of these sensors from GRP78 and their activation, eventually leading to cell death. Therefore, activation of GRP78 is one of the obvious signals in the ER signaling pathway. Based on the key role of GRP78 in regulating ER 
homeostasis, protein folding, and protein assembly and degradation, we focused on its expression and release in relation to apoptosis in U937 cells. As shown in Figure 5, the expression of the GRP78 protein was significant upregulated in response to treatment with oTR. This suggested that oTR induced apoptosis of U937 cells through the ERS pathway.

Differentiation therapies play important roles in leukemia treatment. Differentiation therapies in oncology include changing malignant tumors to curable tumors or terminally differentiated cells that undergo no further proliferation [26]. The induction of cancer cell differentiation and tumor eradication through the inhibition of tumor cell proliferation with anti-cancer agents is important in differentiation therapy. $\mathrm{Cd} 11 \mathrm{~b}$ is a granulocytic differentiation marker of hematopoietic cells [27]. In the present study, we showed that oTR significantly upregulated CD11b expression in U937 cells (Figure 6). Furthermore, Wright-Giemsa staining showed that treatment with oTR reduced the nuclear cytoplasmic ratio and altered the horseshoe morphology of nuclei (Figure 7). These results suggested that oTR promoted the differentiation of U937 cells towards the granulocytic lineage.

In addition to their DNA synthesis directed actions, many nucleoside analogs as anticancer drugs trigger apoptosis by unique mechanisms, such as causing epigenetic modifications [13]. In cancer, epigenetic silencing often occurs by multiple processes. Until recently, epigenetic studies in cancer focused on the aberrant methylation of stretches of cytosine-guanine residues that formed $\mathrm{CpG}$ islands within gene promoters. Because DNMT catalyze the process of DNA methylation, we assessed the DNMT activity and Molecular Modelling treated with oTR in U937cancer cells. Our study found that oTR acted as an effective inhibitor of DNMT1 (Figures 8 and 9).

In conclusion, our results demonstrated that oTR inhibits the proliferation of human AML U937 cells by inducing apoptosis through the ERS pathway and the granulocytic differentiation of U937 cells. Furthermore, our data suggested that oTR has the potential to be a potent therapeutic agent for targeting DNA methylation for the treatment of human acute leukemia, but further evidence of the underlying mechanism is required to confirm our hypothesis.

\section{Conflict of Interest}

All authors declare that they have no conflict of interest.

\section{Acknowledgement}

This work was supported by the Fundamental Research Funds for the Central Universities of China (No. 5006-851008) and National Natural Science Foundation of China (No. 31371431).

\section{References}

1. Hatzimichael E, Georgiou G, Benetatos L, Briasoulis E. Gene mutations and molecularly targeted therapies in acute myeloid leukemia. Am J Blood Res 2013; 3: 29-51.
2. Sook-Kyoung Heo E-KN, Dong-Joon Y, Jae-Cheol J, JaeHoo P, Hawk K. Dasatinib accelerates valproic acidinduced acute myeloid leukemia cell death by regulation of differentiation capacity. PLoS One 2014; 9.

3. Voller J, Zatloukal M, Lenobel R, Dolezal K, Beres T, Krystof V. Anticancer activity of natural cytokinins: A structure-activity relationship study. Phytochemistry 2010; 71: 1350-1359.

4. Zhang ZG, Zou J, Huang Y, Wu L. Kinetin inhibits proliferation of hepatic stellate cells by interrupting cell cycle and induces apoptosis by down-regulating ratio of Bcl-2/Bax. J Huazhong Univ Sci Technol Med Sci 2015; 35: 672-678.

5. Zhao L, Liu P, Guo G, Wang L. Combination of cytokinin and auxin induces apoptosis, cell cycle progression arrest and blockage of the Akt pathway in HeLa cells. Mol Med Rep 2015; 12: 719-727.

6. Ciaglia E, Pisanti S, Picardi P, Laezza C, Sosa S, Tubaro A. N6-isopentenyladenosine affects cytotoxic activity and cytokines production by IL-2 activated NK cells and exerts topical anti-inflammatory activity in mice. Pharm Res 2014; 89: 1-10.

7. Rajabi M, Mehrzad J, Gorincioi E, Santaniello E. Antiproliferative activity of N (6)-isopentenyladenosine on HCT-15 colon carcinoma cell line. Nucleic Acid Therap 2011; 21: 355-358.

8. Laezza C, Malfitano AM, Di Matola T, Ricchi P, Bifulco M. Involvement of Akt/NF-kappaB pathway in N6isopentenyladenosine-induced apoptosis in human breast cancer cells. Mol Carcinogenesis 2010; 49: 892-901.

9. Laezza C, Caruso MG, Gentile T, Notarnicola M, Malfitano AM, Di Matola T. N6-isopentenyladenosine inhibits cell proliferation and induces apoptosis in a human colon cancer cell line DLD1. Int J Cancer 2009; 124: 1322-1329.

10. Ottria R, Casati S, Maier JA, Mariotti M, Ciuffreda P. Novel isopentenyladenosine analogues: synthesis, characterization, and evaluation of antiproliferative activity on bladder carcinoma cells. Nucleosides Nucleotides Nucleic Acids 2009; 28: 736-751.

11. Choi BH, Kim W, Wang QC, Kim DC, Tan SN, Yong JW. Kinetin riboside preferentially induces apoptosis by modulating Bcl-2 family proteins and caspase-3 in cancer cells. Cancer Lett 2008; 261: 37-45.

12. Hewett EW, Wareing PF. Cytokinins in Populus x robusta (schneid): Light effects on endogenous levels. Planta 1973; 114: 119-129.

13. Ewald B, Sampath D, Plunkett W. Nucleoside analogs: molecular mechanisms signaling cell death. Oncogene 2008; 27: 6522-6537.

14. Wang L, Sun C, Wang ZH, Guo GQ. Mechanism of apoptotosis induced by ortho-topolin riboside in human hepatoma cell line SMMC-7721. Food Chem Toxicol 2012; 50: 1962-1968.

15. Dicks N, Gutierrez K, Michalak M, Bordignon V, Agellon LB. Endoplasmic reticulum stress, genome damage, and cancer. Frontiers Oncol 2015; 5: 11. 
16. Kang X, Lu Z, Cui C, Deng M, Fan Y, Dong B. The ITIMcontaining receptor LAIR1 is essential for acute myeloid leukaemia development. Nat Cell Biol 2015; 17: 665-677.

17. Wang M, Wang L, Pan XJ, Zhang H. Monocytic differentiation of K562 cells induced by proanthocyanidins from grape seeds. Archiv Pharm Res 2012; 35: 129-135.

18. Drenichev MS, Oslovsky VE, Mikhailov SN. Cytokinin nucleosides-natural compounds with a unique spectrum of biological activities. Curr Topics Med Chem 2016; 16: 2562-2576.

19. Kim JI, Cho SR, Lee CM, Park ES, Kim KN, Kim HC. Induction of ER stress-mediated apoptosis by alpha-lipoic acid in A549 cell lines. Kor J Thorac Cardiovas Surg 2012; 45: $1-10$.

20. Gupta S. Molecular signaling in death receptor and mitochondrial pathways of apoptosis (Review). Int J Oncol 2003; 22: 15-20.

21. Kadowaki H, Nishitoh H, Ichijo H. Survival and apoptosis signals in ER stress: the role of protein kinases. J Chem Neuroanatomy 2004; 28: 93-100.

22. Saito A. Physiological functions of endoplasmic reticulum stress transducer OASIS in central nervous system. Anatomical Sci Int 2014; 89: 11-20.

23. Kim I, Xu W, Reed JC. Cell death and endoplasmic reticulum stress: disease relevance and therapeutic opportunities. Nat Rev Drug Discov 2008; 7: 1013-1030.
24. Khadir A, Kavalakatt S, Abubaker J, Cherian P, Madhu D, Al-Khairi I. Physical exercise alleviates ER stress in obese humans through reduction in the expression and release of GRP78 chaperone. Metabolism: Clin Exp 2016; 65: 1409-1420.

25. Walter P, Ron D. The unfolded protein response: from stress pathway to homeostatic regulation. Science 2011; 334: 1081-1086.

26. Spira AI, Carducci MA. Differentiation therapy. Curr Opinion Pharmacol 2003; 3: 338-343.

27. Chotirat S, Suriyo T, Hokland M, Hokland P, Satayavivad J, Auewarakul CU. Cholinergic activation enhances retinoic acid-induced differentiation in the human NB-4 acute promyelocytic leukemia cell line. Blood Cells Mol Dis 2016; 59: 77-84.

\section{${ }^{*}$ Correspondence to}

Changhao Cui

School of Life and Medicine

Dalian University of Technology

Pan Jin

Liaoning

P. R China 